\title{
The Role of Cytokines in the Functional Activity of Phagocytes in Blood and Colostrum of Diabetic Mothers
}

\author{
Danny Laura Gomes Fagundes, ${ }^{1}$ Eduardo Luzía França, ${ }^{2}$ \\ Glilciane Morceli, ${ }^{1}$ Marilza Vieira Cunha Rudge, ${ }^{1}$ \\ Iracema de Mattos Paranhos Calderon, ${ }^{1}$ and Adenilda Cristina Honorio-França ${ }^{2}$ \\ ${ }^{1}$ Graduate Program in Gynecology, Obstetrics and Mastology of Botucatu Medical School, \\ São Paulo State University (UNESP), Av. Prof. Montenegro Bairro: Distrito de Rubião Junior, s/n, 18618-970 Botucatu, SP, Brazil \\ ${ }^{2}$ Institute of Biological and Health Science, Federal University of Mato Grosso (UFMT), \\ Rodovia BR070, Km 5 s/n, 78600-000 Barra do Garças, MT, Brazil
}

Correspondence should be addressed to Adenilda Cristina Honorio-França; denifran@terra.com.br

Received 24 October 2013; Revised 17 November 2013; Accepted 17 November 2013

Academic Editor: Sharad Rastogi

Copyright (C) 2013 Danny Laura Gomes Fagundes et al. This is an open access article distributed under the Creative Commons Attribution License, which permits unrestricted use, distribution, and reproduction in any medium, provided the original work is properly cited.

\begin{abstract}
Immune response changes induced by diabetes are a risk factor for infections during pregnancy and may modify the development of the newborn's immune system. The present study analyzed colostrum and maternal and cord blood of diabetic women to determine (1) the levels of the cytokines IFN- $\gamma$ and TGF- $\beta$ and (2) phagocytic activity after incubation with cytokines. Methods. Colostrum and maternal and cord blood samples were classified into normoglycemic $(N=20)$ and diabetic $(N=19)$ groups. Cytokine levels, superoxide release, rate of phagocytosis, bactericidal activity, and intracellular $\mathrm{Ca}^{2+}$ release by phagocytes were analyzed in the samples. Irrespective of glycemic status, IFN- $\gamma$ and TGF- $\beta$ levels were not changed in colostrum and maternal and cord blood. In maternal blood and colostrum, superoxide release by cytokine-stimulated phagocytes was similar between the groups. Compared to spontaneous release, superoxide release was stimulated by IFN- $\gamma$ and TGF- $\beta$ in normoglycemic and diabetic groups. In the diabetic group, cord blood phagocytes incubated with IFN- $\gamma$ exhibited higher phagocytic activity in response to EPEC, and maternal blood exhibited lower microbicidal activity. These data suggest that diabetes interferes in maternal immunological parameters and that IFN- $\gamma$ and TGF- $\beta$ modulate the functional activity of phagocytes in the colostrum, maternal blood, and cord blood of pregnant diabetic women.
\end{abstract}

\section{Introduction}

Maternal interaction with the fetus is bidirectional. Fetal and placental tissues require suitable environment, under homeostasis, whereas the maternal body is affected by factors related to metabolic adjustments. In this relationship, the fetus receives passive immunity from the mother, which is crucial for newborn adaptation to the extrauterine environment because it provides protection against infectious agents during the first months of life $[1,2]$.

Cells with phagocytic and microbicidal activity are among the multiple immune components of blood and human milk that play an important role in child protection $[3,4]$. A number of studies report that diabetic patients have low phagocytic and microbicidal activity and reactive oxygen species production due to changes in their antioxidant systems. Moreover, the reduction in phagocytic and microbicidal activity of leukocytes is likely related to an increase in blood glucose levels [5-7].

In diabetic individuals, the balance between proinflammatory and anti-inflammatory cytokines is not fully understood. Some studies show that they prioritize the production of proinflammatory cytokines [8], whereas others relate that the production of both cytokine types is increased by diabetes [9].

The cytokine gamma interferon (IFN- $\gamma$ ) and transforming growth factor $\beta$ (TGF- $\beta$ ) seem to act in the early stages of pregnancy $[10,11]$ and participate in cellular functions. It 
is believed that IFN- $\gamma$ promotes the inflammatory reaction that enables trophoblast implantation, whereas TGF- $\beta$ acts on maternal immunological response, embryo implantation, and placental and fetal development $[10,12]$.

IFN- $\gamma$ and TGF- $\beta$ play a role in the activation and regulation of immune cells. IFN- $\gamma$ promotes the microbicidal response of phagocytes, increasing the expression of surface receptors and rates of phagocytosis [13]. TGF- $\beta$, in turn, likely induces and maintains the immune tolerance to the fetus [12] and the production of regulatory cells [14]. Nevertheless, the action of these cytokines on the microbicidal activity of blood and colostrum phagocytes of diabetic mothers has yet to be investigated.

The present study analyzed the colostrum and maternal and cord blood of diabetic women in order to determine (1) the levels of the cytokines IFN- $\gamma$ and TGF- $\beta$ and (2) phagocytic activity after incubation with cytokines.

\section{Materials and Methods}

The functional activity of colostrum and maternal and cord blood phagocytes in diabetic women was evaluated in a cross-sectional study. The subjects attended the Diabetes and Pregnancy Facility, School of Medicine Obstetrics Course, UNESP, Botucatu, SP, Brazil. This study was approved by the institutional Research Ethics Committee, and all the subjects gave written informed consent before entering the experimental protocol.

2.1. Subjects. Blood and colostrum samples from pregnant women (18-45 years old) were analyzed by maternal glycemic status. According to the results of the $75 \mathrm{~g}$ oral glucose tolerance test (OGTT $75 \mathrm{~g}$ ) [15] and glucose profile (GP) test [16], 39 pregnant women were classified into the following groups: normoglycemic group (normal $75 \mathrm{~g}$ OGTT and normal GP; $n=20$ ) and diabetes mellitus group (altered GTT $75 \mathrm{~g}$, prior to or during the pregnancy and abnormal GP; $n=$ 19). The subjects continued attending the facility, irrespective of diagnosis, and the diabetic patients followed a specific treatment for glycemic control [16].

2.2. Blood Sampling and Separation of Blood Cells. Samples of $8 \mathrm{~mL}$ of maternal blood were collected prior to the beginning of labor and cord blood at birth in tubes with anticoagulant. We centrifuged them at $160 \mathrm{G}$ for $15 \mathrm{~min}$ to separate plasma from the cells. Cells were separated by a Ficoll-Paque gradient (Pharmacia, Uppsala, Sweden), producing preparations with 95\% of pure mononuclear cells, analyzed by light microscopy. Purified macrophages were resuspended independently in serum-free medium 199 at a final concentration of $2 \times$ $10^{6}$ cells $/ \mathrm{mL}$. The cells were used immediately for assays of superoxide release, phagocytosis, microbicidal activity, and calcium release. The plasma was stored at $-80^{\circ} \mathrm{C}$ for later glucose and cytokines analysis.

2.3. Colostrum Sampling and Separation of Colostral Cells. About $8 \mathrm{~mL}$ of colostrum from each woman was collected in sterile plastic tubes between 48 and 72 hours postpartum. The samples were centrifuged $\left(160 \mathrm{G}, 4^{\circ} \mathrm{C}\right)$ for $10 \mathrm{~min}$, which separated colostrum into three different phases: cell pellet, an intermediate aqueous phase, and a lipid-containing supernatant. The upper fat layer was discarded and the aqueous supernatant was stored at $-80^{\circ} \mathrm{C}$ for later analyses. Cells were separated by a Ficoll-Paque gradient (Pharmacia, Uppsala, Sweden), producing preparations with $98 \%$ of pure mononuclear cells, analyzed by light microscopy. Purified macrophages were resuspended independently in serum-free medium 199 at a final concentration of $2 \times 10^{6}$ cells $/ \mathrm{mL}$. The cells were used for assays of superoxide release, phagocytosis, microbicidal activity, and calcium release. The colostrum supernatant was stored at $-80^{\circ} \mathrm{C}$ for later glucose and cytokines analysis.

2.4. Glucose Determination. Glucose levels were determined by the enzymatic system. Samples of $20 \mu \mathrm{L}$ colostrum/ maternal or cord blood, standard of $100 \mathrm{mg} / \mathrm{dL}$ (Doles), were placed in $2.0 \mathrm{~mL}$ phosphate buffer solution $(0.05 \mathrm{M}$, pH7.45, with aminoantipyrine $0.03 \mathrm{mM}, 15 \mathrm{mM}$ sodium $\mathrm{p}$ hydroxybenzoate, $12 \mathrm{kU} / \mathrm{L}$ glucose oxidase, and $0.8 \mathrm{kU} / \mathrm{L}$ peroxidase). The suspensions were mixed and incubated for $5 \mathrm{~min}$ at $37^{\circ} \mathrm{C}$. The reactions were read on a spectrophotometer at $510 \mathrm{~nm}$.

2.5. Cytokine Dosage By ELISA (Enzyme-Linked Immunosorbent Assay). IFN- $\gamma$ concentrations in the colostrum and milk supernatants were determined by an ELISA kit from BioLegend Legend Max (San Diego, USA), and TGF- $\beta$ concentrations were analyzed using an ELISA kit from Enzo Life Sciences (UK). The reaction rates were measured by absorbance in a spectrophotometer with a $450 \mathrm{~nm}$ filter. The results were calculated using the standard curve and shown in $\mathrm{pg} / \mathrm{dL}$.

2.6. Escherichia Coli Strain. The enteropathogenic Escherichia coli (EPEC) used was isolated from stools of an infant with acute diarrhea (serotype 0111: $\mathrm{H}^{-} A L^{-}, e a e^{+}, e a f^{+}, b f p^{+}$). This material was prepared and adjusted to $10^{7}$ bacteria $/ \mathrm{mL}$, as previously described by Honorio-França [17].

\subsection{Treatment of Blood and Colostral Phagocytes with Cytok-} ines. To assess the effect of cytokines (IFN- $\gamma$ and TGF$\beta$ ) on superoxide anion release, phagocytic, microbicidal activity, and intracellular $\mathrm{Ca}^{2+}$ release, $\mathrm{MN}$ phagocytes $(2 \times$ $10^{6}$ cells $/ \mathrm{mL}$ ) were incubated with $5 \mu \mathrm{L}$ of cytokines (Sigma St. Louis, USA, final concentration $100 \mathrm{ng} / \mathrm{mL}$ ) for $1 \mathrm{~h}$ at $37^{\circ} \mathrm{C}$. The phagocytes were then washed once with 199 medium at $4^{\circ} \mathrm{C}$ and immediately used in the assays. A control was performed with only 199 medium.

2.8. Release of Superoxide Anion. Superoxide release was determined by cytochrome C (Sigma, St. Louis, USA) reduction $[17,18]$. Briefly, mononuclear phagocytes (blood and colostrum) and bacteria were mixed and incubated for 30 min for phagocytosis. Cells were then resuspended in PBS 
TABLE 1: Mean ( \pm SD) glucose level, leukocyte count, viability, IFN- $\gamma$, and TGF- $\beta$ concentrations in colostrum, maternal blood, and cord blood from normoglycemic and diabetic women.

\begin{tabular}{|c|c|c|c|}
\hline Parameter & Sample & Normoglycemic & Diabetic \\
\hline \multirow{3}{*}{ Glucose level (mg/dL) } & Colostrum & $66.0 \pm 7.4$ & $114.2 \pm 9.6^{*}$ \\
\hline & Maternal blood & $90.5 \pm 8.6$ & $122.1 \pm 8.9^{*}$ \\
\hline & Cord blood & $69.0 \pm 7.3$ & $87.0 \pm 8.5^{*}$ \\
\hline \multirow{3}{*}{ Mononuclear phagocytes count $\left(\times 10^{6} \mathrm{cell} / \mathrm{mL}\right)$} & Colostrum & $2.8 \pm 0.5$ & $2.4 \pm 0.8$ \\
\hline & Maternal blood & $4.8 \pm 0.7$ & $5.1 \pm 0.5$ \\
\hline & Cord blood & $2.9 \pm 0.6$ & $3.2 \pm 0.9$ \\
\hline \multirow{3}{*}{ Mononuclear phagocytes viability (\%) } & Colostrum & $92.0 \pm 3.5$ & $90.0 \pm 2.2$ \\
\hline & Maternal blood & $91.0 \pm 2.4$ & $90.0 \pm 3.3$ \\
\hline & Cord blood & $90.0 \pm 2.6$ & $91.0 \pm 2.7$ \\
\hline \multirow{3}{*}{$\mathrm{IFN}-\gamma(\mathrm{pg} / \mathrm{mL})$} & Colostrum & $9.2 \pm 1.5$ & $7.9 \pm 3.1$ \\
\hline & Maternal blood & $8.2 \pm 1.7$ & $8.4 \pm 0.5$ \\
\hline & Cord blood & $8.9 \pm 1.8$ & $7.5 \pm 2.6$ \\
\hline \multirow{3}{*}{ TGF- $\beta(\mathrm{pg} / \mathrm{mL})$} & Colostrum & $22.1 \pm 1.6$ & $24.0 \pm 4.3$ \\
\hline & Maternal blood & $26.1 \pm 4.7$ & $26.6 \pm 3.7$ \\
\hline & Cord blood & $26.0 \pm 4.6$ & $27.6 \pm 4.4$ \\
\hline
\end{tabular}

${ }^{*}$ Statistical differences in glucose levels between normoglycemic and diabetic groups, considering the same kind of samples.

containing $2.6 \mathrm{mM} \mathrm{CaCl}_{2}, 2 \mathrm{mM} \mathrm{MgCl}$, and cytochrome $\mathrm{C}$ (Sigma, St. Louis, USA; $2 \mathrm{mg} / \mathrm{mL})$. The suspensions $(100 \mu \mathrm{L})$ were incubated for $60 \mathrm{~min}$ at $37^{\circ} \mathrm{C}$ on culture plates. The reaction rates were measured by absorbance at $550 \mathrm{~nm}$ and the results were expressed as $\mathrm{nmol} / \mathrm{O}_{2}{ }^{-}$. All the experiments were performed in duplicate.

2.9. Bactericidal Assay. Phagocytosis and microbicidal activity were evaluated by the acridine orange method [19]. Equal volumes of bacteria and cell suspensions were mixed and incubated at $37^{\circ} \mathrm{C}$ for $30 \mathrm{~min}$ under continuous shaking. Phagocytosis was stopped by incubation in ice. To eliminate extracellular bacteria, the suspensions were centrifuged twice $\left(160 \times \mathrm{g}, 10 \mathrm{~min}, 4^{\circ} \mathrm{C}\right)$. Cells were resuspended in serum-free 199 medium and centrifuged. The supernatant was discarded and the sediment was dyed with $200 \mu \mathrm{L}$ of acridine orange (Sigma, St. Louis, USA; $14.4 \mathrm{~g} / \mathrm{L}$ ) for $1 \mathrm{~min}$. The sediment was resuspended in cold 199 medium, washed twice, and observed under immunofluorescence microscope at 400x and 1000x magnification.

The phagocytosis index was calculated by counting the number of cells ingesting at least 3 bacteria in a pool of 100 cells. To determine the bactericidal index, we stained the slides with acridine orange and counted 100 cells with phagocytized bacteria. The bactericidal index is calculated as the ratio between orange-stained (dead) and green-stained (alive) bacteria x100 [4]. All the experiments were performed in duplicate.

2.10. Intracellular $\mathrm{Ca}^{2+}$ Release Determined by Fluorescence and Flow Cytometry. We performed fluorescence staining at the FACS Calibur (BD, San Jose, USA) to assess intracellular $\mathrm{Ca}^{2+}$ release in phagocytes [20]. Cells were loaded with the fluorescent radiometric calcium indicator Fluo3acetoxymethyl (Fluo3-AM-Sigma, St. Louis, USA). Cell suspensions, pretreated or not with $50 \mu \mathrm{L}$ of cytokines (Sigma, final concentration of $100 \mathrm{ng} / \mathrm{mL}$ ), were mixed and incubated at $37^{\circ} \mathrm{C}$ for $30 \mathrm{~min}$ under continuous stirring. Suspensions were centrifuged twice $\left(160 \times \mathrm{g}, 10 \mathrm{~min}, 4^{\circ} \mathrm{C}\right)$ and resuspended in PBS containing BSA $(5 \mathrm{mg} / \mathrm{mL})$. This suspension was incubated with $5 \mu \mathrm{L}$ of Fluo-3 $(1 \mu \mathrm{g} / \mathrm{mL})$ for $30 \mathrm{~min}$ at $37^{\circ} \mathrm{C}$. After incubation, cells were washed twice in PBS containing BSA $\left(5 \mathrm{mg} / \mathrm{mL} ; 160 \times \mathrm{g}, 10 \mathrm{~min}, 4^{\circ} \mathrm{C}\right)$ and then analyzed by flow cytometry (FACS Calibur system, BD, San Jose, USA). Calibration and sensitivity were routinely checked using CaliBRITE 3 Beads (BD, Cat. no 340486, USA). Fluo-3 was detected at 530/30 $\mathrm{nm}$ filter for intracellular $\mathrm{Ca}^{2+}$. The rate of intracellular $\mathrm{Ca}^{2+}$ release was expressed in geometric mean fluorescence intensity of Fluo-3. Data shown in the figures correspond to one of several trials performed.

2.11. Statistical Analysis. Data were expressed as the mean \pm standard deviation (SD). The statistically significant difference was evaluated using the analysis of variance (ANOVA) for superoxide release anion, phagocytosis, bactericidal index, and intracellular $\mathrm{Ca}^{2+}$ release in the presence or absence of cytokines and the difference was compared at the statistical significance was considered for a $P$-value less than 0.05 .

\section{Results}

Glucose levels in colostrum, maternal blood, and cord blood were higher in hyper- than in normoglycemic women. However, leukocyte retrieval and viability and IFN- $\gamma$ and TGF- $\beta$ levels in maternal blood, cord blood, and colostrum samples were similar between the groups (Table 1).

Diabetic and normoglycemic groups had similar spontaneous superoxide release by mononuclear ( $\mathrm{MN}$ ) phagocytes in colostrum. When exposed to EPEC and cytokines, the phagocytes of both groups increased superoxide release $(P<$ $0.05)$. Irrespective of the presence of cytokines, phagocytes 
TABLE 2: Superoxide release by colostrum and mononuclear phagocytes of blood (mean $\pm \mathrm{SD}, N=10$ in each treatment).

\begin{tabular}{|c|c|c|c|}
\hline \multirow{2}{*}{ Phagocytes } & \multirow{2}{*}{ Incubated with } & \multicolumn{2}{|c|}{ Superoxide release $(\mathrm{nmol})$} \\
\hline & & Normoglycemic & Diabetic \\
\hline \multirow{4}{*}{ Colostrum } & PBS & $1.7 \pm 0.4$ & $2.0 \pm 0.5$ \\
\hline & Bacteria & $4.4 \pm 0.8^{*}$ & $4.1 \pm 0.6^{*}$ \\
\hline & Bacteria plus IFN- $\gamma$ & $4.6 \pm 0.5^{*}$ & $3.5 \pm 0.4^{*+}$ \\
\hline & Bacteria plus TGF- $\beta$ & $4.1 \pm 0.5^{*}$ & $3.6 \pm 0.1^{*+}$ \\
\hline \multirow{4}{*}{ Maternal blood } & PBS & $1.8 \pm 0.2$ & $1.7 \pm 0.2$ \\
\hline & Bacteria & $3.5 \pm 0.8^{*}$ & $3.8 \pm 0.4^{*}$ \\
\hline & Bacteria plus IFN- $\gamma$ & $4.1 \pm 0.8^{*}$ & $3.7 \pm 0.4^{*}$ \\
\hline & Bacteria plus TGF- $\beta$ & $4.3 \pm 0.5^{*}$ & $4.5 \pm 0.1^{* \#}$ \\
\hline \multirow{4}{*}{ Cord blood } & PBS & $3.2 \pm 0.5^{\#}$ & $4.9 \pm 1.1^{+\#}$ \\
\hline & Bacteria & $4.8 \pm 0.8^{*}$ & $4.6 \pm 1.0$ \\
\hline & Bacteria plus IFN- $\gamma$ & $5.4 \pm 0.7^{*}$ & $5.1 \pm 0.5^{\#}$ \\
\hline & Bacteria plus TGF- $\beta$ & $4.6 \pm 0.3^{*}$ & $5.6 \pm 0.1^{\#+}$ \\
\hline
\end{tabular}

Colostrum and blood mononuclear cells were treated or not with cytokines, in the presence or absence of EPEC. ${ }^{*}$ Indicates differences between phagocytes treated or not with cytokines and incubated with bacteria and the control (without bacteria) within each group and sample (colostrum, maternal blood, or cord blood); " indicates differences between sample (colostrum, maternal blood, and cord blood) within each treatment (cytokines or PBS) and group; and ${ }^{+}$indicates intergroup differences within each treatment (cytokines or PBS) and sample (colostrum, maternal blood, or cord blood).

in maternal blood showed the highest superoxide release when exposed to bacteria. In the diabetic group, cord blood phagocytes displayed higher spontaneous superoxide release than those in the normoglycemic group. In the normoglycemic group, cord blood phagocytes exhibited the highest superoxide release when exposed to bacteria and cytokines. In the diabetic group, cytokines did not affect superoxide release by cord blood phagocytes (Table 2).

$\mathrm{MN}$ phagocytes from colostrum and maternal blood, treated or not with cytokines, exhibited similar phagocytic activity against EPEC, irrespective of glycemic status (Figure 1). Cord blood phagocytes from the diabetic group displayed a higher phagocytic index when exposed to bacteria and IFN- $\gamma(P<0.05$-Figure 1$)$.

In general, maternal blood phagocytes in the diabetic group showed low bactericidal activity against EPEC. Both groups exhibited equivalent rates of EPEC elimination by mononuclear phagocytes when treated with cytokines. Maternal blood phagocytes, in both the groups studied, treated with IFN- $\gamma$ exhibited a higher bactericidal index. The bactericidal index of colostrum and cord blood phagocytes did not vary between the groups (Figure 2).

In the diabetic group, maternal blood phagocytes had low intracellular $\mathrm{Ca}^{2+}$ release, irrespective of cytokine treatment. In the normoglycemic group, cord blood phagocytes showed lower intracellular $\mathrm{Ca}^{2+}$ release than those in maternal blood. In the presence of TGF- $\beta$, intracellular $\mathrm{Ca}^{2+}$ release was higher in cord cells from the normoglycemic group. In the diabetic group, colostrum phagocytes not incubated with cytokines showed lower intracellular $\mathrm{Ca}^{2+}$ release when compared with cells from normoglycemic group (Table 3 and Figure 3).

\section{Discussion}

The present study describes IFN- $\gamma$ and TGF- $\beta$ levels in colostrum and maternal and cord blood of diabetic women

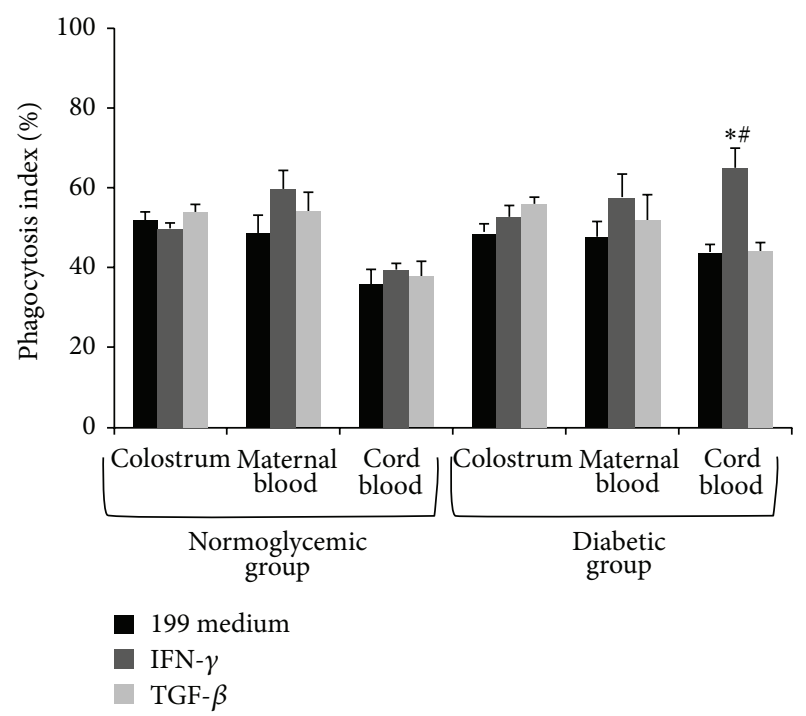

FIGURE 1: Bacterial phagocytosis by colostrum and maternal and cord blood phagocytes (mean $\pm \mathrm{SD}, N=10$ in each treatment and sample), determined by the acridine orange method. Phagocytes were incubated with enteropathogenic Escherichia coli (EPEC) in the presence of gamma interferon (IFN- $\gamma$ ) and transforming growth factor $\beta$ (TGF- $\beta$ ). ${ }^{*}$ indicates differences from the 199 medium and cytokines use within each sample and group; ${ }^{\#}$ indicates differences between normoglycemic and diabetic groups within each treatment (cytokines or 199 medium) and sample (colostrum, maternal blood, or cord blood).

and how these cytokines affect phagocytic activity in the maternal body and the gestation products evaluated.

Several factors affect cytokine production and action during pregnancy [21]. One of these cytokines, IFN- $\gamma$, is implicated in the network of mediators of diabetes [22]. In the present study, however, hyperglycemia did not affect the levels of IFN- $\gamma$ and TGF- $\beta$ in colostrum and maternal and cord blood. 
TABLE 3: Intracellular $\mathrm{Ca}^{2+}$ release by mononuclear (MN) colostrum phagocytes of diabetic mothers indicated by fluorescence intensity.

\begin{tabular}{lcrr}
\hline Phagocytes & Incubated with & \multicolumn{2}{c}{ Fluorescence intensity (\%) } \\
& & Normoglycemic & $17.8 \pm 1.5^{+}$ \\
Colostrum & PBS & $20.2 \pm 0.3$ & $19.6 \pm 5.2$ \\
& IFN- $\gamma$ & $22.0 \pm 2.4$ & $18.1 \pm 3.28$ \\
\hline \multirow{3}{*}{ Maternal blood } & TGF- $\beta$ & $20.4 \pm 0.56$ & $9.6 \pm 2.3^{\#+}$ \\
& PBS & $14.2 \pm 2.3^{\#}$ & $10.2 \pm 2.2^{\#+}$ \\
& IFN- $\gamma$ & $16.6 \pm 2.6^{\#}$ & $9.1 \pm 2.6^{\#+}$ \\
Cord blood & TGF- $\beta$ & $15.5 \pm 3.8^{\#}$ & $10.8 \pm 1.4^{\#}$ \\
& PBS & $10.2 \pm 2.1^{\#}$ & $10.9 \pm 1.4^{\#}$ \\
\hline
\end{tabular}

Colostrum and blood mononuclear cells were preincubated or not with cytokines. ${ }^{*}$ Indicates differences between phagocytes incubated with cytokines and the control (PBS) within each group and sample, ${ }^{\#}$ indicates differences between samples (colostrum, maternal blood, and cord blood) within each treatment (cytokines or PBS) and group; and ${ }^{+}$indicates differences between normo- and diabetic groups within each treatment (cytokines or PBS) and sample (colostrum, maternal blood, or cord blood).

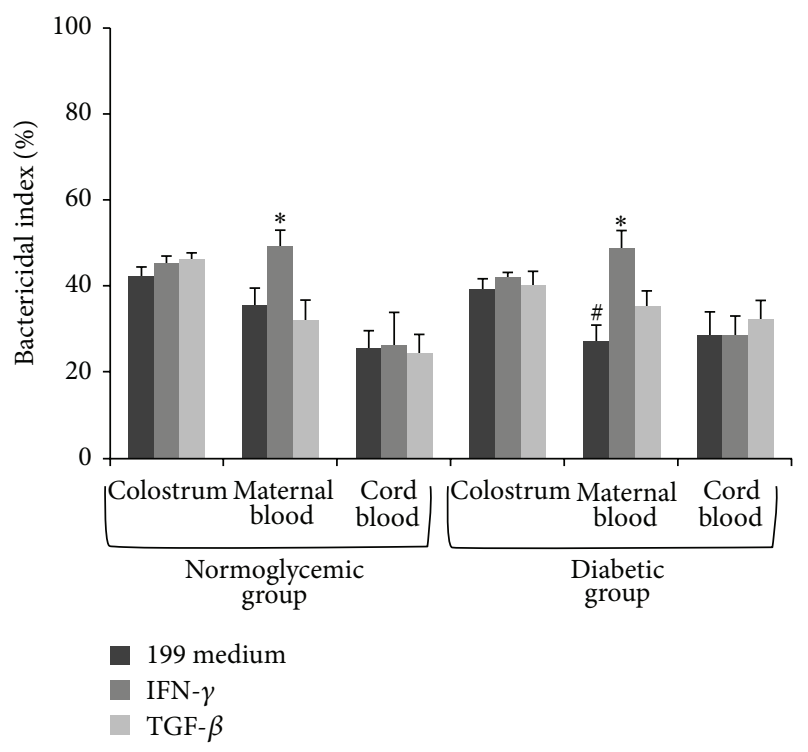

Figure 2: Bactericidal index (mean $\pm \mathrm{SD}, N=10$ in each treatment and sample), determined by the acridine orange method. Phagocytes were incubated with enteropathogenic Escherichia coli (EPEC) in the presence of gamma interferon (IFN- $\gamma$ ) and transforming growth factor $\beta$ (TGF- $\beta$ ). * Indicates differences from the 199 medium and cytokines use within each sample and group; \#indicates differences between normoglycemic and diabetic groups within each treatment (cytokines or 199 medium) and sample (colostrum, maternal blood, or cord blood).

A number of studies show that part of the clinical picture of diabetic patients is associated with excessive release of proinflammatory cytokines. Cytokines exert profound effects on the biological signaling and regulation of important physiological processes that are compromised by diabetes [22]. Cytokines may also be related to phagocyte activation and production of reactive oxygen species [23].

In the present study, the cytokines tested modulated superoxide release. Colostrum phagocytes and maternal blood phagocytes increased superoxide release in both groups studied. On the other hand, in the diabetic group, cord blood phagocytes exhibited the highest spontaneous superoxide release, suggesting phagocyte activation irrespective of cytokine presence. A number of mechanisms possibly contribute to the formation of these reactive oxygen-free radicals. Glucose oxidation is believed to be the main source of free radicals [24]. Hyperglycemia also promotes lipid peroxidation by a superoxide-dependent pathway, producing free radicals $[25,26]$. Another important source of free radicals in diabetic individuals is the products of glucoseprotein interaction [27].

The functional activity of phagocytes was assessed in the colostrum and blood samples of diabetic patients $[1,3,7,28]$ and animals with induced diabetes $[29,30]$. Phagocytes play an important role in host defense. Here, we showed that, in the presence of cytokines, phagocytes from colostrum and maternal blood exhibit similar phagocytic activity against EPEC, irrespective of the women's glycemic status. In the diabetic group, cord blood phagocytes increase phagocytosis rate in the presence of IFN- $\gamma$.

Phagocytosis and microbicidal activity of phagocytes in colostrum and blood, with production of active oxygen metabolites such as free radicals, consist of an important defense mechanism against a number of bacterial [4, 31], fungal [32], and protozoal infections [33]. In the present study, we found that phagocytes in the blood of diabetic mothers exhibited low bactericidal activity against EPEC. However, after incubation with cytokines, bacterial elimination by $\mathrm{MN}$ phagocytes of diabetic women increased to rates similar to those obtained in the normoglycemic group.

Earlier studies have reported that diabetic patients have low phagocytic and microbicidal activity due to derangements in prooxidative systems. An increase in blood glucose levels is indeed related to a decrease in the phagocytic activity of leukocytes [5-7]. On the other hand, cytokines such as IFN- $\gamma$ act primarily on macrophages by activating their phagocytic and microbicidal abilities $[13,34]$. The present study is the first to report the effects of IFN- $\gamma$ and TGF- $\beta$ on the functional activity of colostrum and maternal and cord blood phagocytes of diabetic mothers. 

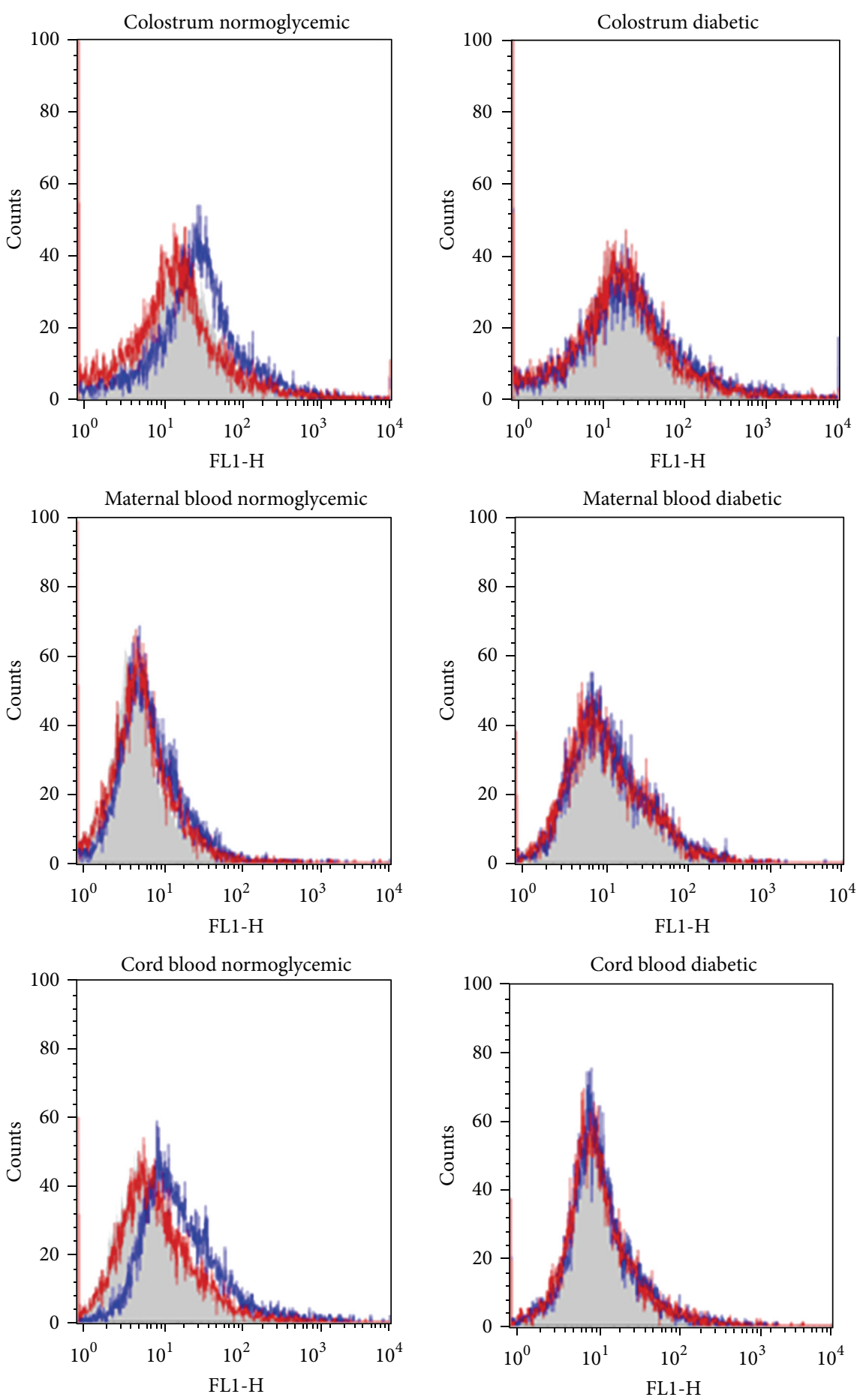

Phagocytes

Phagocytes + IFN- $\gamma$

Phagocytes + TGF- $\beta$

Phagocytes

Phagocytes + IFN- $\gamma$

Phagocytes + TGF- $\beta$

FIGURE 3: Intracellular $\mathrm{Ca}^{2+}$ release by colostral, maternal blood and cord blood phagocytes of diabetic mothers stimulated or not with cytokines. Cells were stained with Fluo-3, and immunofluorescence analyses were carried out by flow cytometry (FACScalibur, Becton Dickinson, USA). 
Interestingly, cord blood phagocytes exposed to EPEC and stimulated by IFN- $\gamma$ showed an increase in phagocytosis but not in their bactericidal activity. A number of studies report that these cells display low bactericidal activity because they lack nonspecific surface receptors, while others argue that they are still too immature to exhibit this response [1].

In diabetic patients, cytokine production may be associated with a number of processes such as alterations in intracellular $\mathrm{Ca}^{+2}$ by phagocytes, which is triggered by advanced glycation and products [35] and hyperglycemia [36]. In the present study, cytokines did not stimulate intracellular $\mathrm{Ca}^{2+}$ release by maternal blood phagocytes in the diabetic group, and in the normoglycemic group, TGF- $\beta$ increased intracellular $\mathrm{Ca}^{2+}$ release in cord cells.

Transforming growth factor $\beta$ (TGF- $\beta$ ) is involved in the balance of maternal immune response and deployment process and produced early in the maternal-fetal interface by the embryo and decidua $[10,14]$.

Although colostral phagocytes of diabetic women decrease intracellular $\mathrm{Ca}^{2+}$ release in relation to normoglycemic women [7], we show here that colostrum phagocytes, irrespective of cytokine incubation, increase intracellular $\mathrm{Ca}^{2+}$ release in relation to maternal and cord blood phagocytes.

The maternal transfer of immune components during pregnancy and breastfeeding represents a remarkable immunologic interaction between the mother and newborn. Breast milk is an excellent source of immunological components, and it decreases the high rates of maternal and infant complications. Adequate maternal glycemic control during pregnancy and breastfeeding duration in diabetic mothers is crucial to ensure that the immunity components act against the infections.

\section{Conclusion}

Our findings support the hypothesis that diabetes interferes in maternal immunological parameters due to alterations in glucose metabolism and that IFN- $\gamma$ and TGF- $\beta$ can modulate the functional activity of colostrum, maternal blood, and cord blood phagocytes of diabetic women and these cytokines can be alternative for use in future clinical applications in diabetic patients.

\section{Conflict of Interests}

The authors declare no conflict of interests and nonfinancial competing interests regarding the publication of this paper.

\section{Acknowledgments}

The authors are very grateful to the Diabetes and Pregnancy Service, Obstetrics Discipline of Botucatu Medical School, UNESP. This research was supported by FAPESP (no. 201118045-5; no. 2011-23671-2), CNPq (no. 475739/2011-6), and CAPES.

\section{References}

[1] E. L. Franca, I. M. P. Calderon, E. L. Vieira, G. Morceli, and A. C. Honorio-França, "Transfer of maternal immunity to newborns of diabetic mothers," Clinical Development Immunology, vol. 2012, Article ID 928187, 7 pages, 2012.

[2] G. Morceli, E. L. França, V. B. Magalhães, D. C. Damasceno, I. M. P. Calderon, and A. C. Honorio-França, "Diabetes induced immunological and biochemical changes in human colostrum," Acta Paediatrica, vol. 100, no. 4, pp. 550-556, 2011.

[3] E. L. Franca, T. D. R. Nicomedes, I. D. M. P. Calderon, and A. C. H. Franca, "Time-dependent alterations of soluble and cellular components in human milk," Biological Rhythm Research, vol. 41, no. 5, pp. 333-347, 2010.

[4] E. L. França, R. V. Bitencourt, M. Fujimori, T. Cristina de Morais, I. de Mattos Paranhos Calderon, and A. C. HonorioFrança, "Human colostral phagocytes eliminate enterotoxigenic Escherichia coli opsonized by colostrum supernatant," Journal of Microbiology, Immunology and Infection, vol. 44, no. 1, pp. 1-7, 2011.

[5] T. C. Alba-Loureiro, C. D. Munhoz, J. O. Martins et al., "Neutrophil function and metabolism in individuals with diabetes mellitus," Brazilian Journal of Medical and Biological Research, vol. 40, no. 8, pp. 1037-1044, 2007.

[6] E. L. França, G. Morceli, D. L. G. Fagundes, M. V. C. Rudge, I. de Mattos Paranhos Calderon, and A. C. Honorio-França, "Secretory IgA-Fc $\alpha$ receptor interaction modulating phagocytosis and microbicidal activity by phagocytes in human colostrum of diabetics," Acta Pathologica, Microbiologica et Immunologica Scandinavica, vol. 119, no. 10, pp. 710-719, 2011.

[7] G. Morceli, A. C. Honorio-França, D. L. G. Fagundes, I. M. P. Calderon, and E. L. Franca, "Antioxidant effect of melatonin on the functional activity of colostral phagocytes in diabetic women," PLoS ONE, vol. 8, no. 2, Article ID e56915, 2013.

[8] J. Chen and X. S. Liu, "Development and function of IL-10 IFN$\gamma$-secreting CD $4^{+}$T cells," Journal of Leukocyte Biology, vol. 86, no. 6, pp. 1305-1310, 2009.

[9] A. Chatzigeorgiou, V. Harokopos, C. Mylona-Karagianni, E. Tsouvalas, V. Aidinis, and E. Kamper, "The pattern of inflammatory/anti-inflammatory cytokines and chemokines in type 1 diabetic patients over time," Annals of Medicine, vol. 42, no. 6, pp. 426-438, 2010.

[10] S. A. Robertson, W. V. Ingman, S. O’Leary, D. J. Sharkey, and K. P. Tremellen, "Transforming growth factor $\beta$-a mediator of immune deviation in seminal plasma," Journal of Reproductive Immunology, vol. 57, no. 1-2, pp. 109-128, 2002.

[11] G. Chaouat, “The Th1/Th2 paradigm: still important in pregnancy?" Seminars in Immunopathology, vol. 29, no. 2, pp. 95-113, 2007.

[12] A. A. Ashkar, G. P. Black, Q. Wei et al., "Assessment of requirements for IL-15 and IFN regulatory factors in uterine NK cell differentiation and function during pregnancy," Journal of Immunology, vol. 171, no. 6, pp. 2937-2944, 2003.

[13] S. Casares and T.-D. Brumeanu, "Insights into the pathogenesis of type 1 diabetes: a hint for novel immunospecific therapies," Current Molecular Medicine, vol. 1, no. 3, pp. 357-378, 2001.

[14] V. R. Aluvihare, M. Kallikourdis, and A. G. Betz, "Regulatory T cells mediate maternal tolerance to the fetus," Nature Immunology, vol. 5, no. 3, pp. 266-271, 2004.

[15] American Diabetes Association, "Diagnosis and classification of diabetes mellitus," Diabetes Care, vol. 35, pp. S64-S71, 2012. 
[16] M. V. C. Rudge, I. D. M. P. Calderon, M. D. Ramos, J. F. Abbade, and L. M. S. S. Rugolo, "Perinatal outcome of pregnancies complicated by diabetes and by maternal daily hyperglycemia not related to diabetes: a retrospective 10-year analysis," Gynecologic and Obstetric Investigation, vol. 50, no. 2, pp. 108-112, 2000.

[17] A. C. Honorio-França, M. P. S. M. Carvalho, L. Isaac, L. R. Trabulsi, and M. M. S. Carneiro-Sampaio, "Colostral mononuclear phagocytes are able to kill enteropathogenic Escherichia coli opsonized with colostral IgA," Scandinavian Journal of Immunology, vol. 46, no. 1, pp. 59-66, 1997.

[18] E. Pick and D. Mizel, "Rapid microassays for the measurement of superoxide and hydrogen peroxide production by macrophages in culture using an automatic enzyme immunoassay reader," Journal of Immunological Methods, vol. 46, no. 2, pp. 211-226, 1981.

[19] A. C. Honorio-França, C. C. P. Hara, J. V. S. Ormonde, G. T. Nunes, and E. L. França, "Human colostrum melatonin exhibits a day-night variation and modulates the activity of colostral phagocytes," Journal of Applied Biomedicine, vol. 11, pp. 153-162, 2013.

[20] D. L. G. Fagundes, E. L. Franca, C. C. P. Hara, and A. C. Honorio-França, "Immunomodulatory effects of poly (ethylene glycol) microspheres adsorbed with cortisol on activity of colostrum phagocytes," International Journal of Pharmacology, vol. 8, pp. 510-518, 2012.

[21] C. P. Gomes, M. R. Torloni, B. Y. Gueuvoghlanian-Silva, S. M. Alexandre, R. Mattar, and S. Daher, "Cytokine levels in gestational diabetes mellitus: a systematic review of the literature," American Journal of Reproductive Immunology, vol. 69, pp. 545557, 2013.

[22] G. Matarese, C. Procaccini, and V. de Rosa, "At the crossroad of T cells, adipose tissue, and diabetes," Immunology Review, vol. 249, pp. 116-134, 2012.

[23] R. Miesel, R. Hartung, and H. Kroeger, "Priming of NADPH oxidase by tumor necrosis factor alpha in patients with inflammatory and autoimmune rheumatic diseases," Inflammation, vol. 20, no. 4, pp. 427-438, 1996.

[24] M. Dizdaroglu, P. Jaruga, M. Birincioglu, and H. Rodriguez, "Free radical-induced damage to DNA: mechanisms and measurement," Free Radical Biology and Medicine, vol. 32, no. 11, pp. 1102-1115, 2002.

[25] Z.-Y. Jiang, A. C. S. Woollard, and S. P. Wolff, "Hydrogen peroxide production during experimental protein glycation," FEBS Letters, vol. 268, no. 1, pp. 69-71, 1990.

[26] E. C. Tsai, I. B. Hirsch, J. D. Brunzell, and A. Chait, "Reduced plasma peroxyl radical trapping capacity and increased susceptibility of LDL to oxidation in poorly controlled IDDM," Diabetes, vol. 43, no. 8, pp. 1010-1014, 1994.

[27] M. Kawamura, J. W. Heinecke, and A. Chait, "Pathophysiological concentrations of glucose promote oxidative modification of low density lipoprotein by a superoxide-dependent pathway," Journal of Clinical Investigation, vol. 94, no. 2, pp. 771-778, 1994.

[28] A. J. Rassias, C. A. S. Marrin, J. Arruda, P. K. Whalen, M. Beach, and M. P. Yeager, "Insulin infusion improves neutrophil function in diabetic cardiac surgery patients," Anesthesia and Analgesia, vol. 88, no. 5, pp. 1011-1016, 1999.

[29] E. L. França, N. D. Feliciano, K. A. Silva, C. K. Bucalen Ferrari, and A. C. Honorio-França, "Modulatory role of melatonin on superoxide release by spleen macrophages isolated from alloxan-induced diabetic rats," Bratislava Medical Journal, vol. 110, no. 9, pp. 517-522, 2009.
[30] A. C. Honório-França, C. M. F. Marins, F. Boldrini, and E. L. França, "Evaluation of hypoglicemic activity and healing of extract from amongst bark of "Quina do Cerrado" (Strychnos pseudoquina ST. HILL)," Acta Cirurgica Brasileira, vol. 23, no. 6, pp. 504-510, 2008.

[31] M. M. S. Carneiro-Sampaio, M. D. L. M. Da Silva, S. B. Carbonare et al., "Breast-feeding protection against enteropathogenic Escherichia coli," Revista de Microbiologia, vol. 26, pp. 151-154, 1996.

[32] E. Kühlwein and M. Irwin, "Melatonin modulation of lymphocyte proliferation and Th1/Th2 cytokine expression," Journal of Neuroimmunology, vol. 117, no. 1-2, pp. 51-57, 2001.

[33] A. C. Franca-Botelho, J. L. Franca, F. M. S. Oliveira et al., "Melatonin reduces the severity of experimental amoebiasis," Parasites \& Vectors, vol. 4, pp. 62-67, 2011.

[34] W. Y. Almawi, H. Tamim, and S. T. Azar, "Clinical review 103: $\mathrm{T}$ helper type 1 and 2 cytokines mediate the onset and progression of type I (insulin-dependent) diabetes," Journal of Clinical Endocrinology and Metabolism, vol. 84, no. 5, pp. 14971502, 1999.

[35] K. S. Collison, R. S. Parhar, S. S. Saleh et al., "RAGE-mediated neutrophil dysfunction is evoked by Advanced Glycation End Products (AGEs)," Journal of Leukocyte Biology, vol. 71, no. 3, pp. 433-444, 2002.

[36] T. M. Demerdash, N. Seyrek, M. Smogorzewski, W. Marcinkowski, S. Nasser-Moadelli, and S. G. Massry, "Pathways through which glucose induces a rise in $\left[\mathrm{Ca}_{2}+\right] \mathrm{i}$ of polymorphonuclear leukocytes of rats," Kidney International, vol. 50, no. 6, pp. 2032 2040, 1996. 


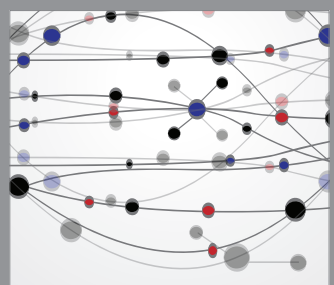

The Scientific World Journal
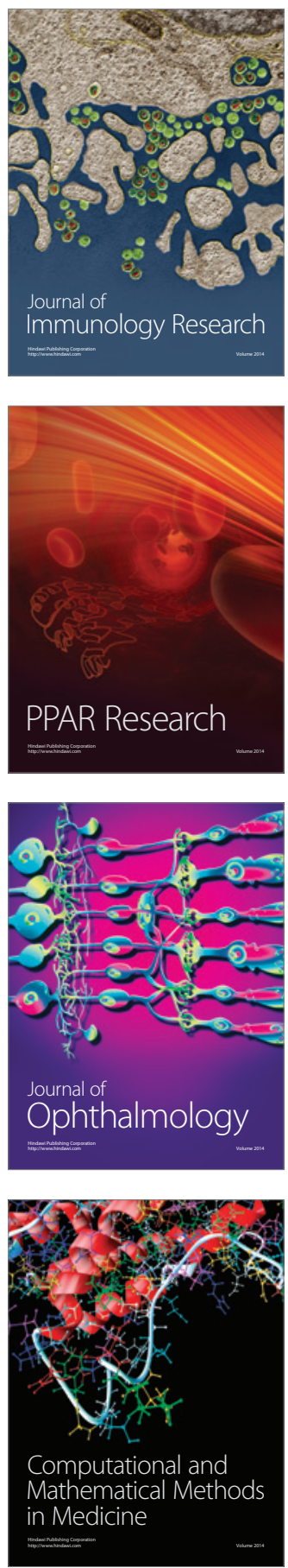

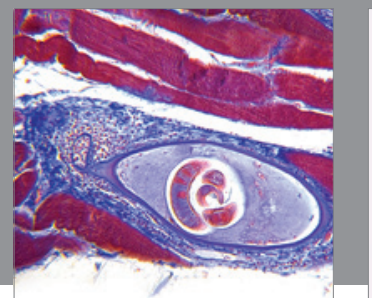

Gastroenterology

Research and Practice
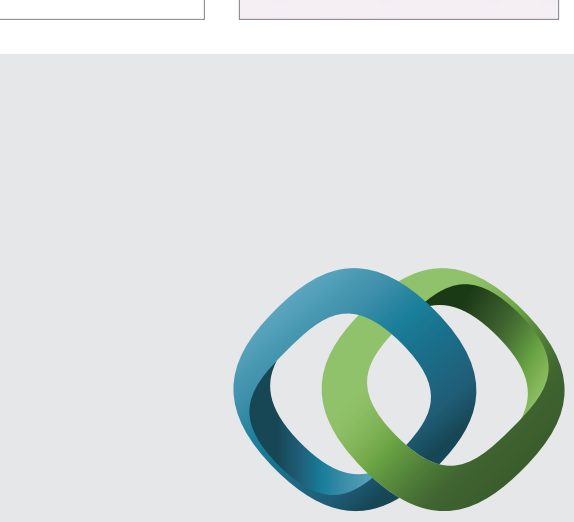

\section{Hindawi}

Submit your manuscripts at

http://www.hindawi.com
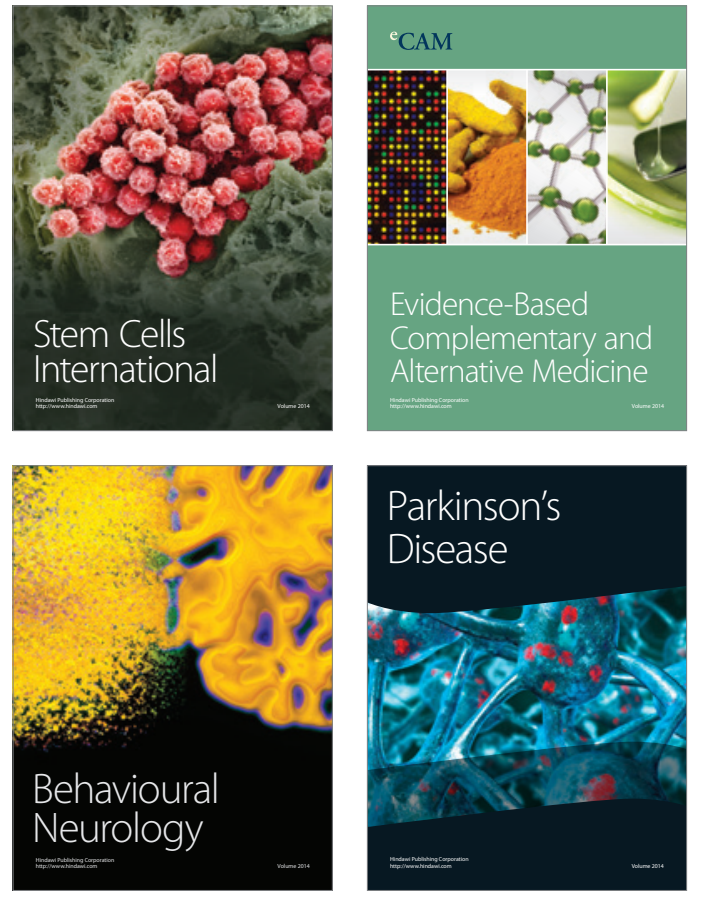
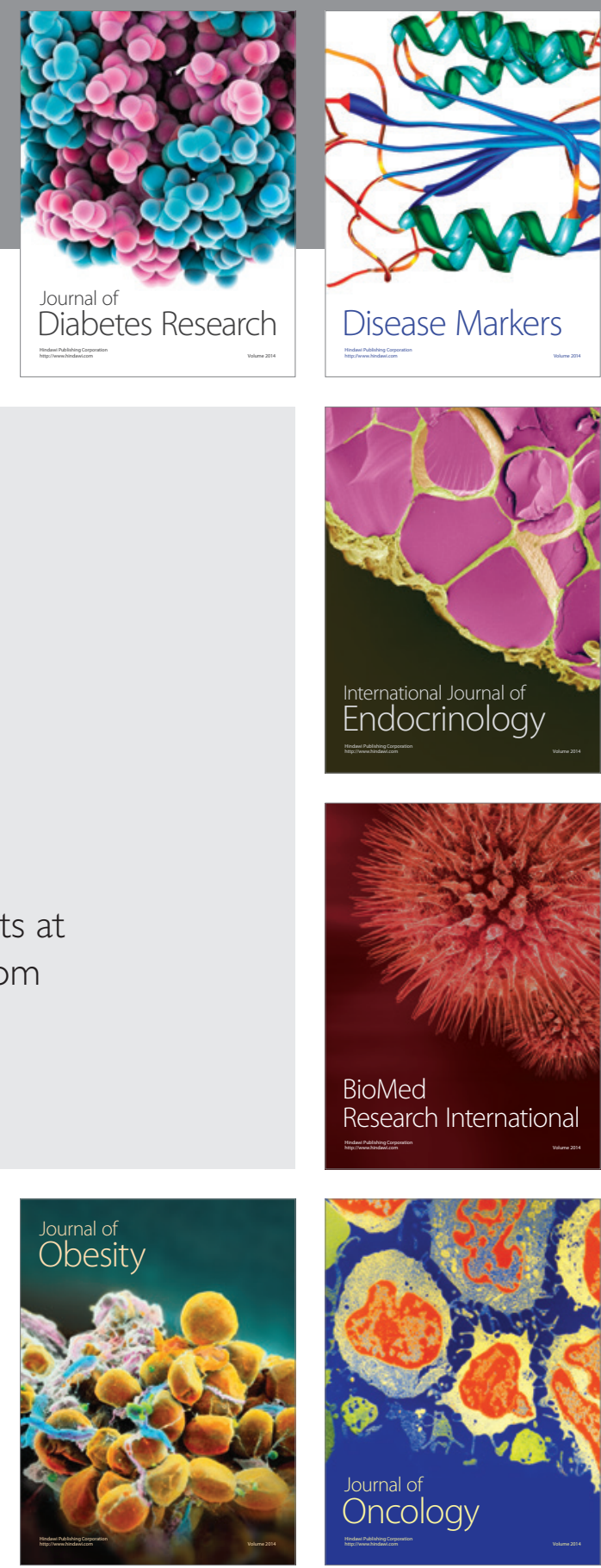

Disease Markers
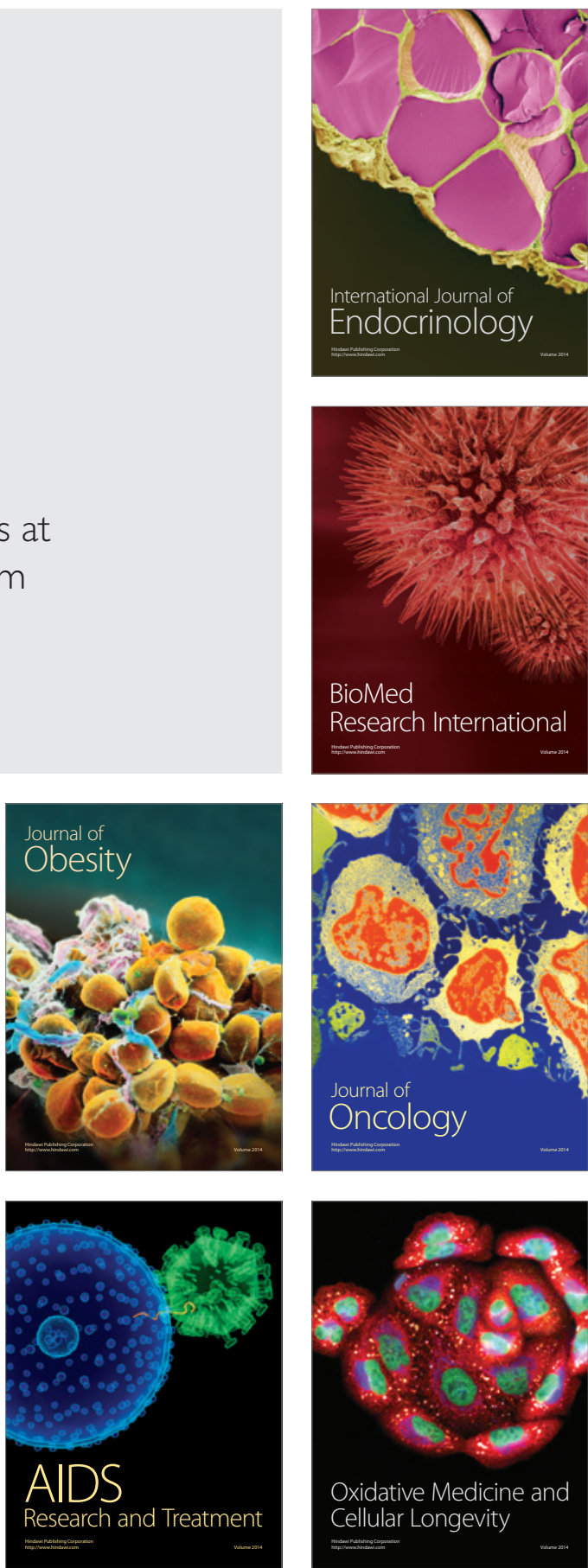